\title{
Spinocerebellar ataxia type 23
}

INSERM

\section{Source}

INSERM. (1999). Orphanet: an online rare disease and orphan drug data base.

Spinocerebellar ataxia type 23. ORPHA:101108

Spinocerebellar ataxia type 23 (SCA23) is a very rare subtype of type I autosomal

dominant cerebellar ataxia (ADCA type I; see this term). It is characterized by gait ataxia,

dysarthria, slowed saccades, ocular dysmetria, Babinski sign and hyperreflexia. 